\title{
ON SAND RIPPLE DETECTION IN SYNTHETIC APERTURE SONAR IMAGERY
}

\author{
David P. Williams and Enrique Coiras \\ NATO Undersea Research Centre \\ Viale San Bartolomeo 400, 19126 La Spezia (SP), Italy
}

\begin{abstract}
A model for the detection of sand ripples in synthetic aperture sonar (SAS) imagery is proposed. The approach is based on searching for patterns characterized by three highlight-shadow pairs - corresponding to ripple-wave crests and troughs - at different orientations and at different length-scales. The model also provides an estimate of the orientation of any ripples detected. No training data is required as the underlying physical phenomenon of ripples is modeled directly. The promise of the proposed method is demonstrated on five real, measured SAS images, for which a high probability of (ripple) detection is achieved while maintaining a very low false alarm rate.
\end{abstract}

Index Terms - Sand ripple detection, synthetic aperture sonar (SAS), ripple model, segmentation, mine countermeasures

\section{INTRODUCTION}

Synthetic aperture sonar (SAS) can provide high-resolution imaging of underwater environments that is particularly useful for mine countermeasure (MCM) operations. Typically, an autonomous underwater vehicle (AUV) will be deployed in MCM operations to survey an area of seabed in a pre-programmed fashion. Ideally, however, the AUV would be allowed to react to information that it collects during the mission by adapting its route so that the most useful data can be collected.

It has been shown [1] that the ability to detect mines in areas of seabed characterized by sand ripples is very difficult (e.g., because targets can be concealed in the shadows of ripples). However, seabed characterized by sand ripples exhibits a strong orientation dependence; when the sand ripple "waves" propagate in the along-track direction of the vehicle (which is assumed to be orthogonal to the look-direction of the sonar), the seabed appears to be flat. Example real SAS imagery illustrating this phenomenon is shown in Fig. 1.

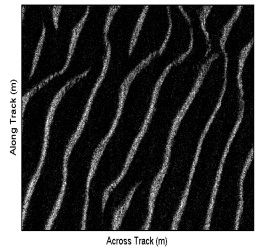

(a)

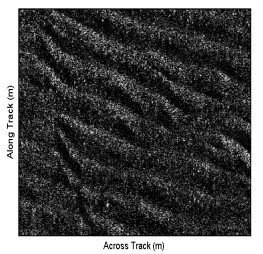

(b)
Fig. 1. SAS imagery of the same area of seabed characterized by ripples when the along-track direction of the vehicle is (a) nearly perpendicular to the ripple propagation direction, and (b) nearly parallel to the ripple propagation direction.

Therefore, if the presence and orientation of sand ripples can be detected and estimated, an AUV can adapt its route so that the seabed is re-inspected at a more favorably heading (namely, one for which the rippled seabed instead appears flat). In turn, the ability to detect mines in that area of seabed should improve substantially.

The limited previous work focusing on ripple detection includes [2] and [3]. In [2], the work did not deal with ripple detection in imagery per se, but rather used high-frequency backscattering data to estimate characteristics of sand ripples.

In [3], ripple detection was attempted using two different approaches, one based on estimating the frequency content of the image and one based on the direction of edges in the image. Both approaches, however, required the ad hoc setting of several parameters with the aid of sets of training data. Moreover, the approach was evaluated mainly on synthetic imagery and could classify only entire images as being rippled or not (i.e., the method could not make distinctions that only certain portions of the image were rippled).

In our work, we avoid using training data - and the adverse implications associated with its use, such as covariate shift (e.g., [4]) - by directly modeling the physical phenomenon of sand ripples. Therefore, the ripple detection approach proposed here is independent of data set and can be reliably employed at any site. Additionally, this work is the first to rely exclusively on high-resolution SAS imagery.

The remainder of this paper is organized in the following manner. The proposed sand ripple model is described in Sec. 2. Experimental results of the approach on real, measured SAS images are shown in Sec. 3, before concluding remarks are made in Sec. 4.

\section{SAND RIPPLE MODEL}

Define the angular increment to be $\Delta=90^{\circ} / K$, where $K>1$ is the number of unique ripple orientations to be considered. The set of "primary" ripple orientations to be considered is then $\boldsymbol{\theta}=\left\{0^{\circ}, \Delta, 2 \Delta, \cdots, K \Delta\right\}$.

Let $\boldsymbol{\lambda}$ be the set of $L$ unique ripple length-scales (i.e., periods) to be considered. For each of the length-scales, $\lambda_{\ell} \in \lambda$, create a filter bank in the following manner.

A base filter is defined such that it exhibits three highlightshadow pairs characteristic of the crests and troughs of a sinusoid. (Three pairs are used because a single highlight-shadow pair can be produced by an elongated object, and two highlight-shadow pairs can be caused by an object in a scour pit.) A rotation, $\theta_{k} \in \boldsymbol{\theta}$, is then applied to produce each of the primary filters. Additionally, for each primary filter, a secondary filter - orthogonal to the primary filter - is created by applying the rotation $\theta_{k}+90^{\circ}$.

An example set of (primary and secondary) filters for the case of $K=3$ is shown in Fig. 2.

Next, upper and lower bounds are calculated for each of the $L$ filter sets being considered (i.e., at each of the $L$ ripple length-scales, $\left.\lambda_{\ell} \in \boldsymbol{\lambda}\right)$. The upper bound, $\beta_{1}\left(\lambda_{\ell}\right)$, is obtained by measuring the filter response to an image of ideal (perfect) ripples. The lower bound, 
Fig. 2. Example set of filters when $K=3$.

$\beta_{0}\left(\lambda_{\ell}\right)$, is obtained by measuring the filter response to a random image with structural elements of the size of the ripples being considered. It must be emphasized that these bounds, $\beta_{1}$ and $\beta_{0}$, are calculated independent of the SAS image being investigated.

Finally, the filter responses to the actual SAS image under investigation are computed. For the $k$-th pair of primary and secondary filters at the $\ell$-th length-scale, the measure of the "ripplicity" is defined to be

$$
\chi\left(\theta_{k}, \lambda_{\ell}\right)=\frac{|| \psi\left(\theta_{k}, \lambda_{\ell}\right)|-| \psi\left(\theta_{k}+90^{\circ}, \lambda_{\ell}\right)||-\beta_{0}\left(\lambda_{\ell}\right)}{\beta_{1}\left(\lambda_{\ell}\right)-\beta_{0}\left(\lambda_{\ell}\right)},
$$

where $\psi\left(\theta_{k}, \lambda_{\ell}\right)$ and $\psi\left(\theta_{k}+90^{\circ}, \lambda_{\ell}\right)$ are the responses with the $\ell$-th length-scale's $k$-th primary and secondary filters, respectively. (The difference of the responses of a pair of primary and secondary filters is used because true ripples should exhibit a particularly lower response in the direction orthogonal to the ripples.)

For each pixel in the image, the maximum ripplicity score computed over each pair of filters in $\boldsymbol{\theta}$ and at each ripple length-scale in $\lambda$, denoted

$$
\rho=\max _{\theta_{k} \in \boldsymbol{\theta}, \lambda_{\ell} \in \boldsymbol{\lambda}}\left\{\chi\left(\theta_{k}, \lambda_{\ell}\right)\right\},
$$

is retained as the final score indicating the degree to which a pixel is part of a ripple. It is this score that is subsequently used for classification purposes.

For each pixel, the orientation $\theta_{k}$ that results in the maximum ripplicity score is also noted. This "map" of orientations is then averaged (over areas on the order of the ripple length-scale used) to obtain the final ripple orientation estimates.

\subsection{Area Filtering}

The ripple model provides a continuous ripplicity score, $\rho$ from (2), for each pixel in the SAS image. What is desired, however, is a binary map indicating disjoint ripple regions and non-ripple regions. To effect such a map, pixels whose ripplicity score is above a fixed threshold, $\tau$, are classified as belonging to ripple areas; that is, the $i j$-th pixel of the image is classified as

$$
y(i, j)= \begin{cases}1, & \text { if } \rho(i, j)>\tau \\ 0, & \text { if } \rho(i, j) \leq \tau\end{cases}
$$

Upon performing this thresholding, however, many small spurious regions in the image may be classified as ripples. A simple way to remove these undesired detections is to filter out any ripple region that contains less than a certain area, $\alpha$,

$$
y^{\prime}(i, j)= \begin{cases}1, & \text { if } y(i, j)=1 \text { and } A(i, j) \geq \alpha ; \\ 0, & \text { otherwise }\end{cases}
$$

where $A(i, j)$ is the area of the ripple region to which the $i j$-th pixel belongs.

The justification for this area-filtering procedure is that one is, in general, not interested in very small ripple regions in an image because the adaptation of the AUV route would be considered only for relatively large ripple regions. That is, the expected benefit of altering a route would exceed the various costs involved only if the ripple region was sufficiently large.

\section{EXPERIMENTAL RESULTS}

\subsection{Data Set}

In April-May 2008, the NATO Undersea Research Centre (NURC) conducted the Colossus II sea trial in the Baltic Sea off the coast of Latvia. During this trial, high-resolution sonar data was collected by the MUSCLE autonomous underwater vehicle (AUV). This AUV is equipped with a $300 \mathrm{kHz}$ sonar with a $60 \mathrm{kHz}$ bandwidth that can achieve an along-track image resolution of approximately $3 \mathrm{~cm}$ and an across-track image resolution of approximately $2.5 \mathrm{~cm}$. The sonar data was subsequently processed into SAS imagery.

We assess the proposed ripple-detection algorithm on five SAS images from this data set. It should be noted that no ripples are present in the fifth image; this case is included to ensure that the approach can succeed even when no ripples are present.

For these experiments, we consider $K=3$ orientations at $L=6$ length-scales, from $0.5 \mathrm{~m}$ to $3 \mathrm{~m}$ at $0.5 \mathrm{~m}$ increments. This set of orientations spans the space of ripple orientations sufficiently (i.e., every $30^{\circ}$ ) while keeping computational costs low. These lengthscales were chosen because they are on the order of the size of targets (i.e., mines) that we are interested in detecting (and thus could be concealed by ripples).

\subsection{Results}

The five SAS images considered in this study are shown in Fig. 3, along with the results of the ripple model in terms of the ripple detection score (from (2)) and estimated ripple orientation.

To obtain the binary (ripple) classification map, we set $\tau=0$ in (3), so that any indication of the presence of a ripple is translated into a ripple detection. The result with additional area filtering by setting $\alpha=25 \mathrm{~m}^{2}$ in (4) is also considered. The results of the ripple detection algorithm in terms of these two binary classification maps are shown in Fig. 4, along with the manually ground-truthed SAS images, for the five images considered.

The probabilities of (ripple) detection and false alarm with $\tau=0$ in (3), both without and with area filtering, are also summarized in Table 1. As can be seen from the table, a very high probability of detection is achieved with a low probability of false alarm. Moreover, the inclusion of the area filtering removes almost all false alarms.

Table 1. Ripple detection performance when $\tau=0$ in (3) and $\alpha=25 \mathrm{~m}^{2}$ in (4).

\begin{tabular}{|c||c|c||c|c|}
\hline \multicolumn{1}{|c||}{ IMAGE } & \multicolumn{2}{c|}{ W/O AREA FILTERING } & \multicolumn{2}{c|}{ W/ AREA FILTERING } \\
\cline { 2 - 5 } Number & $P_{D}$ & $P_{F A}$ & $P_{D}$ & $P_{F A}$ \\
\hline \hline 1 & 0.8175 & 0.0639 & 0.8082 & 0.0111 \\
\hline 2 & 0.7918 & 0.0396 & 0.7717 & 0.0062 \\
\hline 3 & 0.9429 & 0.0705 & 0.9362 & 0.0102 \\
\hline 4 & 0.9768 & 0.0809 & 0.9768 & 0.0183 \\
\hline 5 & 1.0000 & 0.1309 & 1.0000 & 0.0000 \\
\hline
\end{tabular}

\section{CONCLUSIONS}

An elegantly simple model for the detection of sand ripples in SAS imagery was proposed. Importantly, no training data is required because the physical phenomenon of ripples is modeled directly. Moreover, it was observed that the ripple-detection algorithm can handle the case in which no ripples are present in an image. 


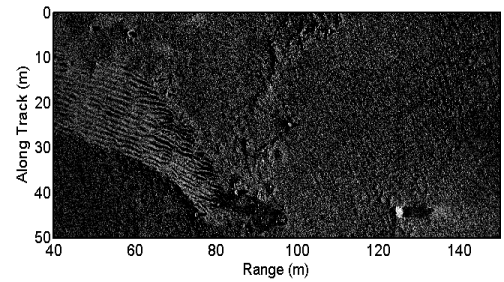

(a) SAS Image 1

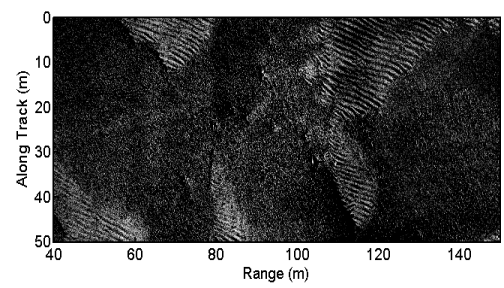

(d) SAS Image 2

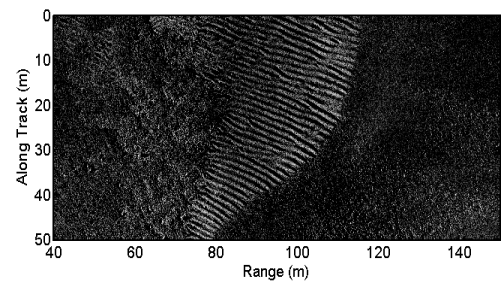

(g) SAS Image 3

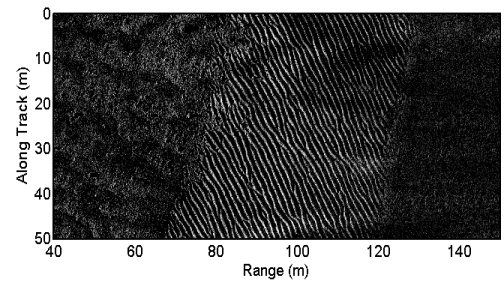

(j) SAS Image 4

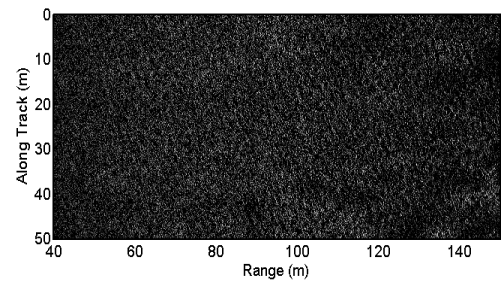

(m) SAS Image 5

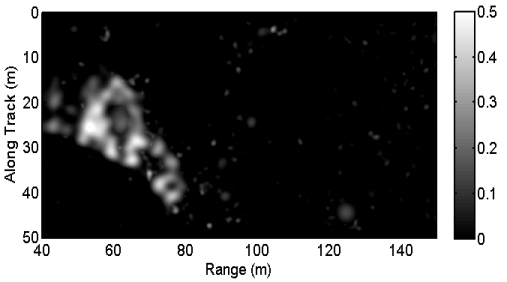

(b) Ripple Detection Score

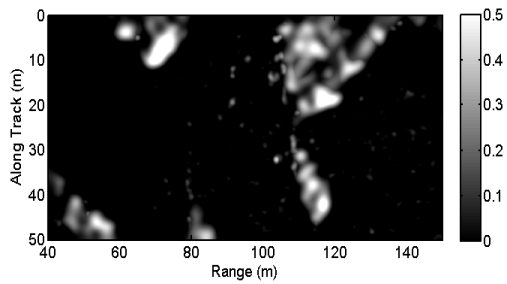

(e) Ripple Detection Score

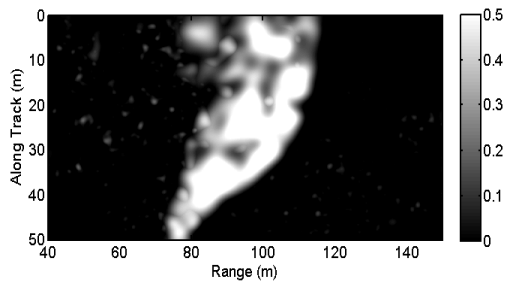

(h) Ripple Detection Score

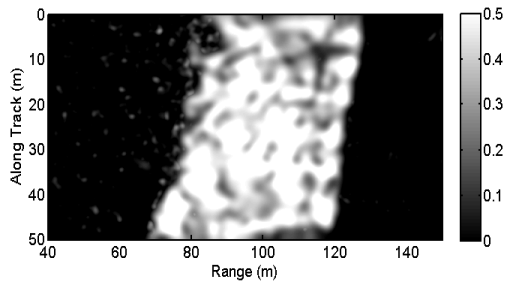

(k) Ripple Detection Score

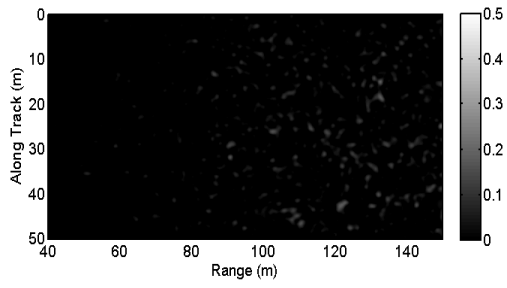

(n) Ripple Detection Score

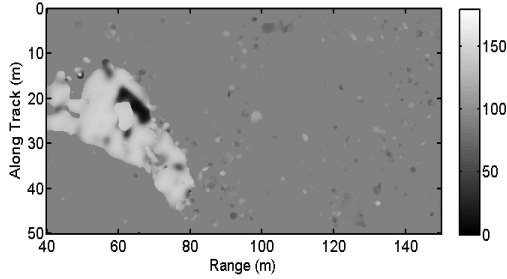

(c) Estimated Ripple Orientation

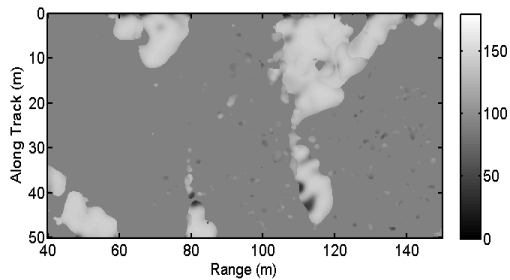

(f) Estimated Ripple Orientation

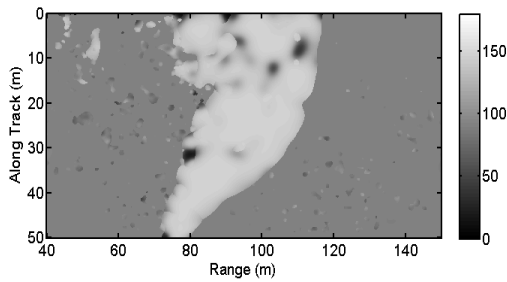

(i) Estimated Ripple Orientation

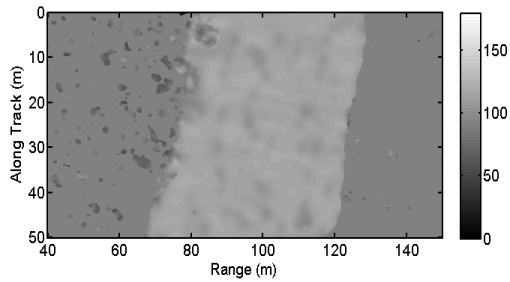

(1) Estimated Ripple Orientation

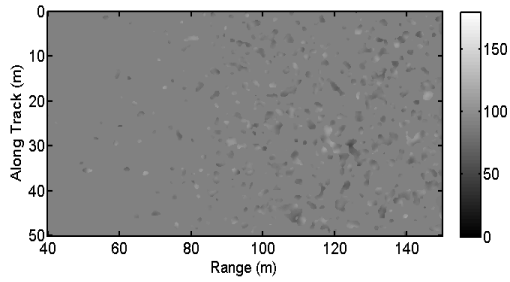

(o) Estimated Ripple Orientation

Fig. 3. Each row of figures shows the SAS image, ripple detection score, and estimated ripple orientation (in degrees, measured from the horizontal), for one of the five SAS images.

This algorithm can be exploited to ensure that the most useful data (for mine-detection purposes) is collected at sea. Specifically, the route of an AUV would be adapted according to the presence and orientation of sand ripples so that the most valuable data - in which sand ripples would appear as flat seabed (cf. Fig. 1) - could be collected.

The proposed ripple-detection algorithm has also been applied with similar success to a much larger data set of thousands of SAS images, not shown here due to space constraints.

\section{REFERENCES}

[1] J. Groen, E. Coiras, and D. Williams, "Detection rate statistics in synthetic aperture sonar images," in Proc. Underwater Acoustic 


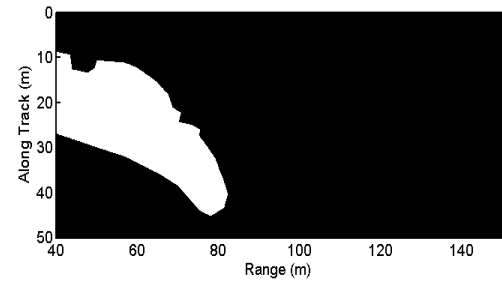

(a) Image 1 Ground Truth

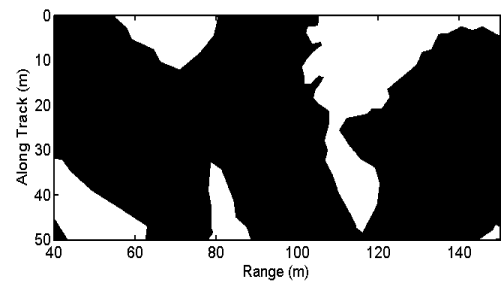

(d) Image 2 Ground Truth

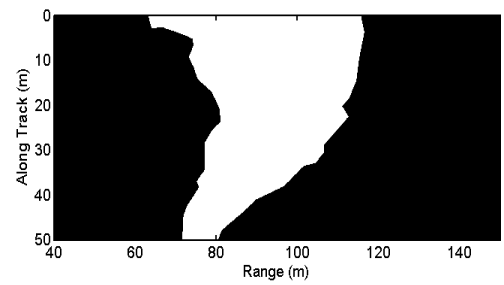

(g) Image 3 Ground Truth

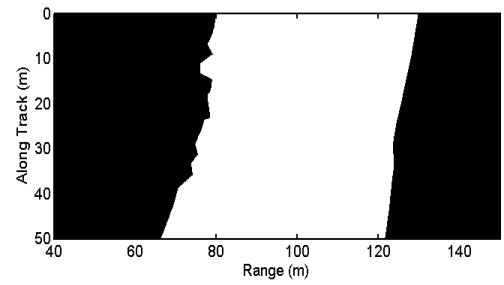

(j) Image 4 Ground Truth

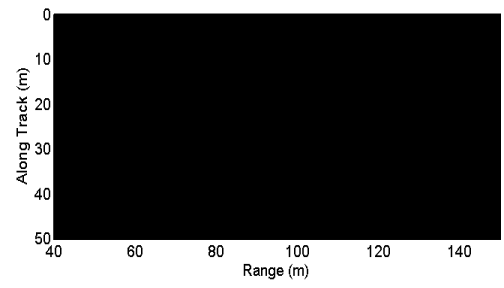

(m) Image 5 Ground Truth

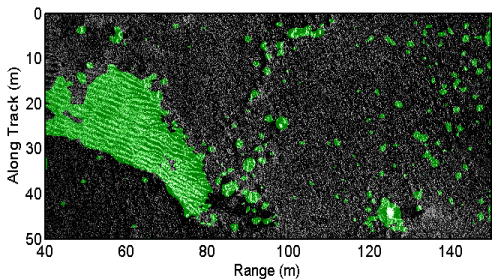

(b) Detection Result Without Area Filtering

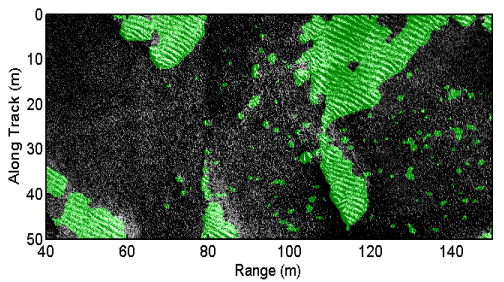

(e) Detection Result Without Area Filtering

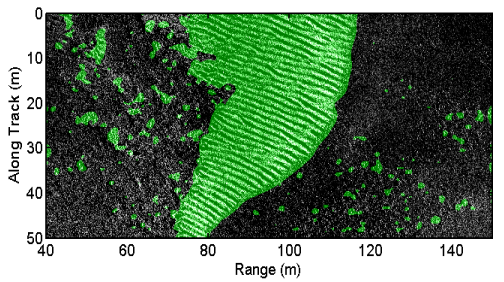

(h) Detection Result Without Area Filtering

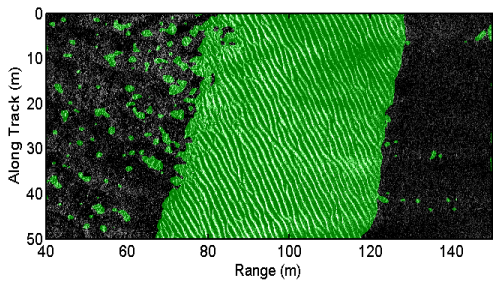

(k) Detection Result Without Area Filtering

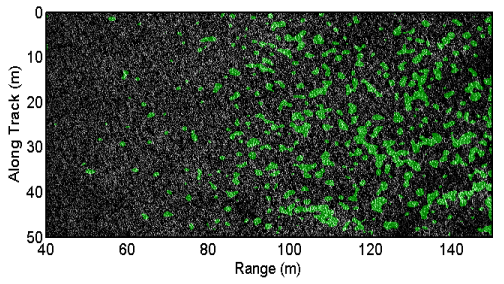

(n) Detection Result Without Area Filtering

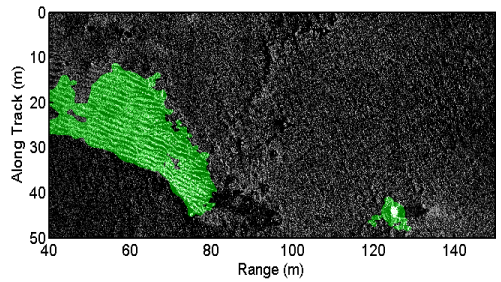

(c) Detection Result With Area Filtering

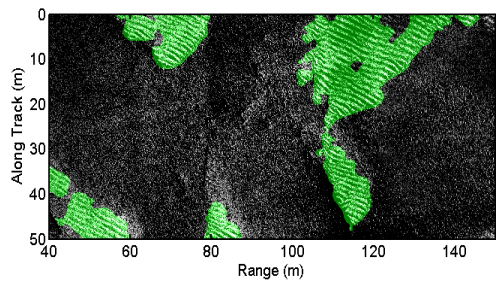

(f) Detection Result With Area Filtering

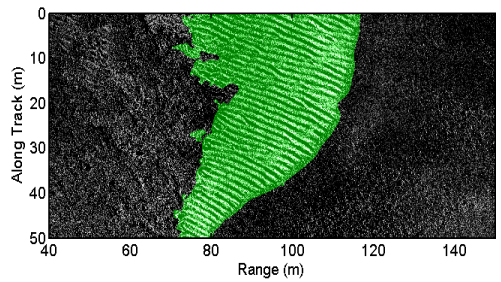

(i) Detection Result With Area Filtering

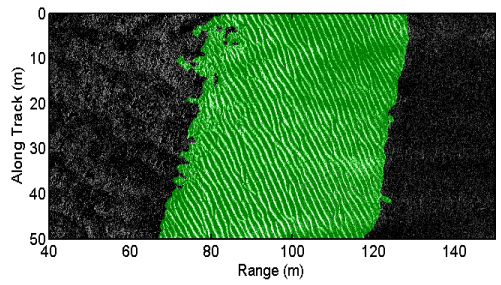

(1) Detection Result With Area Filtering

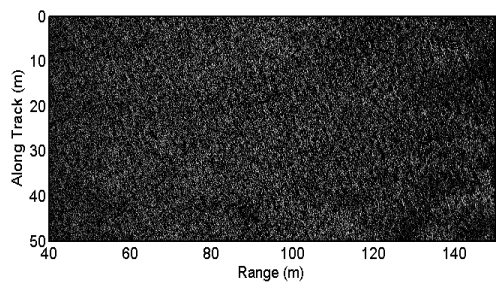

(o) Detection Result With Area Filtering

Fig. 4. Each row of figures shows the ripple ground truth (ripple areas in white), ripple detection result (overlaid on the SAS image) without area filtering, and ripple detection result (overlaid on the SAS image) with area filtering, for one of the five SAS images, when $\tau=0$ in (3) and $\alpha=25 \mathrm{~m}^{2}$ in (4). Regions in green are classified as ripples.

Measurements (UAM), 2009, pp. 367-374.

[2] D. Tang, K. Williams, E. Thorsos, and K. Briggs, "Remote sensing of sand ripples using high-frequency backscatter," in Proc. MTS/IEEE OCEANS, 2002, pp. 2081-2085.

[3] A. Olmos, M. Trucco, K. Lebart, and D. Lane, "Detecting ripple patterns in mission videos," in Proc. MTS/IEEE OCEANS, 2000, pp. 331-335.

[4] M. Sugiyama, M. Krauledat, and K. Müller, "Covariate shift adaptation by importance weighted cross validation," J. Mach. Learn. Res., vol. 8, pp. 985-1005, 2007. 